\title{
PROYECTILES Y PROCESOS DE INTENSIFICACIÓN: UNA APROXIMACIÓN DESDE BOYO PASO 2, CA. 1500-750 AP (SIERRAS DE CÓRDOBA, ARGENTINA)
}

\author{
PROJECTILES AND INTENSIFICATION PROCESSES: AN APPROACH FROM \\ BOYO PASO 2 CA. 1500-750 BP (SIERRAS DE CÓRDOBA, ARGENTINA)
}

\author{
Matías E. Medina ${ }^{1,2}$ Imanol Balena $^{3}$ y Diego E. Rivero ${ }^{1,4}$
}

Se presentan los resultados obtenidos del análisis tecno-tipológico efectuado sobre las puntas de proyectil recuperadas en el sitio Boyo Paso 2 (1500-750 AP, Sierras de Córdoba, Argentina), intentando establecer cómo la dinámica socioeconómica tardía se reflejó en el diseño de los artefactos. Los cabezales fueron analizados consignando atributos tipológico-descriptivos, clasificados en subgrupos tipológicos y finalmente adscritos funcionalmente. El subgrupo tipológico de puntas de proyectil con pedúnculo diferenciado, aletas entrantes y limbo triangular corto de tipo isósceles dominó el conjunto, siendo la calcedonia y el ópalo la roca seleccionada para su elaboración. También se documentaron puntas de proyectil de dimensiones ligeramente mayores en cuarzo, con limbo triangular y base escotada, así como fragmentos asignados a puntas de proyectil de hueso con pedúnculo aserrado y aletas entrantes. La diversidad de cabezales recuperados, junto con los distintos sistemas de enmangue, la adopción generalizada del arco como forma de propulsión, el empleo selectivo de rocas y la incorporación de materias primas óseas, es interpretada como un indicador arqueológico de que la caza no era una actividad complementaria. Por el contrario, integraría junto al cultivo y la recolección un sistema económico de tipo mixto, en donde la flexibilidad fue uno de los rasgos definitorios.

Palabras claves: Sierras de Córdoba, periodo Prehispánico Tardío, puntas de proyectil, sistemas de armas, amplitud del nicho económico.

This paper presents the techno-typological analysis carried out on the projectile points of Boyo Paso 2 (1500-750 year BP, Sierras of Córdoba, Argentina), in order to assess how the dynamic of the late prehispanic sociocultural process influenced the design of hunting weapons. Projectile points were described in techno-typological terms, classified in typological subgroups and functionally assigned to arrow or dart point. The subgroup of tiny arrowpoints with short triangular-shaped blade, contracted stems and barbed shoulders dominates the assemblage. All of them were made of opal and chalcedony, a high quality rock for tool knapping. Moreover, a subgroup of quartz unstemmed with a triangular-shaped blade and concave base dartpoints as well as another subgroup of bone arrowpoints with triangular-shaped blade, straight stems and barbed shoulders were also recognized. The diversity of projectile point-types and hafting methods identified in Boyo Paso 2, along with the extensive use of the bow, the selection of high-quality lithic raw material and the incorporation of bone-tipped projectiles, led to interpret that hunting was not a complementary subsistence activity. Instead, it was integrated in a mixed foraging and cultivation economy where flexibility was one of its defining traits.

Key words: Sierras of Córdoba, Late Pre-Hispanic Period, projectile points, weapon system, niche breadth.

Las puntas de proyectil recuperadas en sitios arqueológicos suelen ser los únicos elementos que se conservaron de los sistemas técnicos en los que estuvieron enmangados (Lyman et al. 2009). En términos generales, se corresponden con un grupo tipológico que formó parte de un artefacto compuesto (arco y flecha, propulsor, entre otros) que sirve para la defensa y el ataque, provocando la muerte de una presa

\footnotetext{
${ }^{1}$ Consejo Nacional de Investigaciones Científicas y Técnicas (CONICET)

${ }^{2}$ División Arqueología, Facultad de Ciencias Naturales y Museo, Universidad Nacional de La Plata, Argentina. paleomedina@ gmail.com

${ }^{3}$ Facultad de Filosofía y Letras, Universidad de Buenos Aires, Púan 480, Buenos Aires (1420), Argentina. Imanol.balena@ gmail.com

${ }^{4}$ Instituto de Estudios Históricos/Centro de Estudios Históricos "Prof. Carlos S.A. Segreti" y Facultad de Filosofía y Humanidades (Universidad Nacional de Córdova), Argentina. ayampitin1@yahoo.com.ar
} 
animal o de una persona a causa de una hemorragia masiva (Christenson 1997; Hughes 1998; Loendorf et al. 2015). En las últimas décadas su estudio ha recibido un creciente interés, destacándose en Argentina los análisis dirigidos a determinar la funcionalidad de las puntas de proyectil, sus procesos de manufactura y reactivación, diseño y relación con las condiciones en las que operaban (Aschero y Martínez 2001; Banegas et al. 2014; Castro 2017; Escola 1991, 2002, 2014; Hocsman 2010; Martínez y Bozzuto 2011; Moreno 2011; Ratto 1989, 2013; Restifo 2013; Sacur Silvestre et al. 2013; Vigna et al. 2014; Weitzel et al. 2014).

En lo que hace específicamente a las Sierras de Córdoba (Argentina), las puntas de proyectil líticas y óseas son artefactos cuya presencia en los contextos asignados al período Prehispánico Tardío (1500-360 AP) resulta llamativa no solo por su significativa representación proporcional, sino también por su diversidad morfológica indicando que ocuparon un lugar significativo dentro de las estrategias tecnológicas. Sin embargo, el análisis de las características de sus diseños y otros aspectos tecnológicos potencialmente vinculados al desempeño funcional quedaron relegadas por la prioridad de atribuir identidad cultural a los contextos y/o estimar su cronología relativa (Berberián 1984; González 1943; González y Crivelli 1978; Marcellino et al. 1967; Serrano 1945), siendo excepcionales los casos en los que se derivaron hipótesis sobre la funcionalidad de las mismas para la realización de prácticas de caza, actividades de defensa/ataque y/o su relación con la estructura regional de recursos (Pastor et al. 2005; Pautassi y Rivero 1999; Rivero y Recalde 2011).

A los efectos de profundizar la discusión sobre los tipos de armas utilizados y el rol funcional que cumplieron dentro de las estrategias de los grupos tardíos, en este trabajo se presentan los resultados obtenidos del análisis tecno-tipológico efectuado sobre el conjunto de puntas de proyectil recuperadas en las excavaciones de Boyo Paso 2 (Sierras de Córdoba, Argentina), un sitio a cielo abierto ocupado en reiteradas ocasiones durante el período Prehispánico Tardío (ca. 1500-750 AP) por grupos que combinaban cultivos, caza-recolección y un uso flexible del espacio. El estudio tuvo como objetivo caracterizar la diversidad tecno-tipológica de los cabezales de armas obtenidos durante los trabajos de campo, intentando establecer las actividades realizadas en el sitio y cómo la dinámica socioeconómica tardía, básicamente asociada a la necesidad de aumentar la productividad total por unidad de tiempo y espacio, se reflejó en el diseño de los artefactos. La variabilidad y alto grado de formatización de las puntas de proyectil, en un contexto dominado por estrategias tecnológicas expeditivas (sensu Nelson 1991), dan particular relevancia a los resultados, en especial para comprender la amplitud del nicho en casos donde la adopción de cultivos no necesariamente derivó en una agricultura sedentaria, un patrón observado etnográficamente, pero difícil de delimitar desde la cultura material.

\section{El Sitio}

BoyoPaso 2(31'19'3,96"'S, 6459' 43,16"O, 1160 $\mathrm{msm}$ ) es un asentamiento a cielo abierto localizado en el sector oriental del valle de Salsacate, Provincia de Córdoba (Figura 1). El entorno inmediato es de Chaco Serrano (Luti et al. 1979), con amplias posibilidades para la recolección de frutos silvestres y suelos potencialmente cultivables restringidos por lomadas rocosas. Las excavaciones realizadas en el sitio revelaron pisos de ocupación asociados a una gran diversidad restos arqueológicos, incluyendo adornos, estatuillas antropomorfas y las puntas de proyectil aquí analizadas, que fueron datados en $750 \pm 70 \mathrm{AP}$, LP2932 (carbón), 866 \pm 39 AP, AA110929 (Phaseolus vulgaris), 870 \pm 50 AP, LP-3577 (carbón), 878 \pm 18 AP,

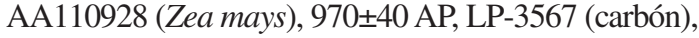
$1060 \pm 50$ AP, LP-3122 (carbón) y 1500 \pm 80 AP, LP310 (carbón). Los conjuntos artefactuales líticos y óseos estaban dominados por instrumentos expeditivos manufacturados con rocas disponibles localmente -i.e. andesitas- y subproductos de las actividades de subsistencia (Medina et al. 2016; 2018). La diversidad taxonómica y el tamaño de los conjuntos faunísticos analizados hasta el momento, dominados por restos óseos de camélidos, cérvidos y pequeños vertebrados, junto la alta densidad de restos cerámicos y líticos recuperados en estratigrafía, son indicativos de estadías de varios meses en el sitio, con una alta incidencia de actividades cinegéticas (Medina et al. 2019). Los indicadores faunísticos de estacionalidad (i.e. huesos de Tupinambis sp., osteodermos de Euphractinae y cáscaras de huevos de Rheidae), junto con evidencias de actividades agrícolas (macro-restos de Z. mays, Phaseolus spp., entre otros) y de recolección (Oxalis sp., Ziziphus mistol, y otros), sugieren que la ocupación del asentamiento coincidió con la primavera-verano, momento del año en que debía realizarse la siembra, cosecha y/o los recursos silvestres estaban disponibles en los alrededores del sitio (Medina et al. 2016; 2018). Por otro lado, la existencia de estructuras habitacionales se infirió a partir de la presencia de numerosos agujeros de postes (Figura 2). Sin embargo, las viviendas no parecen haber sido de tipo permanentes, sino similares a las utilizadas por grupos etnográficos o arqueológicos que cambian estacionalmente de residencia (Medina et al. 2016). Incluso los agujeros de postes, al casi superponerse, desarrollar diámetros variables y al no tener un patrón claro en cuanto a su disposición, pueden representar distintos eventos ocupacionales. El rango de variación de los fechados obtenidos, 


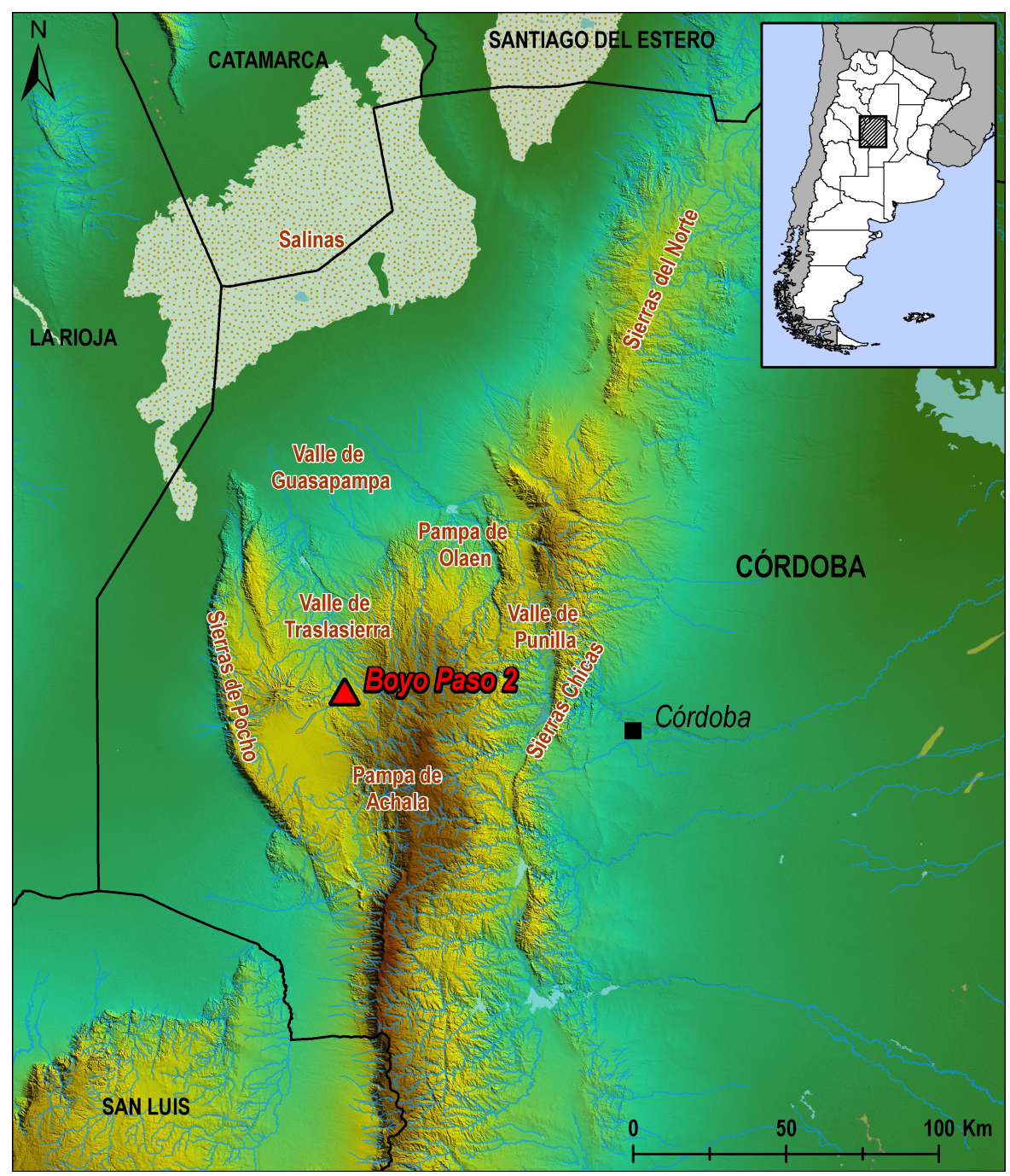

Figura 1. Ubicación geográfica del sitio arqueológico Boyo Paso 2.

Geographic location of the archaeological site Boyo Paso 2.

sumado a conjuntos polínicos dominados por plantas herbáceas comúnmente descritas como indicadoras de un continuo disturbio antrópico, refuerzan este planteo (Medina et al. 2016; 2017). En consecuencia, Boyo Paso 2 es interpretado como un palimpsesto formado por ocupaciones estivales de unas pocas familias para realizar actividades diversas propias de los espacios residenciales, en el marco de una estrategia económica mixta que combinaba cultivos, caza-recolección junto con un uso estacional y diversificado del paisaje.

\section{Materiales y Métodos}

La muestra de puntas de proyectil de Boyo Paso 2 consiste en 38 artefactos, entre preformas $(\mathrm{N}=4)$, piezas enteras $(N=6)$ y fragmentadas $(N=28)$, siendo la clase instrumental más representada en el sitio. La mayoría de las puntas de proyectil provienen de estratigrafía $(\mathrm{N}=34)$, estando asociadas a pisos de ocupación y rasgos de viviendas. Solo cuatro ejemplares se obtuvieron de recolecciones superficiales.

El conjunto fue analizado siguiendo los lineamientos propuestos por Aschero $(1975,1983)$, Hocsman (2010) y Knecht (1993), consignando los siguientes atributos morfológicos-descriptivos: procedencia, dimensiones, serie técnica, formas base, materia prima ${ }^{1}$, sección transversal, ángulo estimado o medido para el ápice, bordes y aletas, forma geométrica, forma de los lascados, morfología de los bordes y bases, sección del filo y peso. Para el abordaje de las variables 


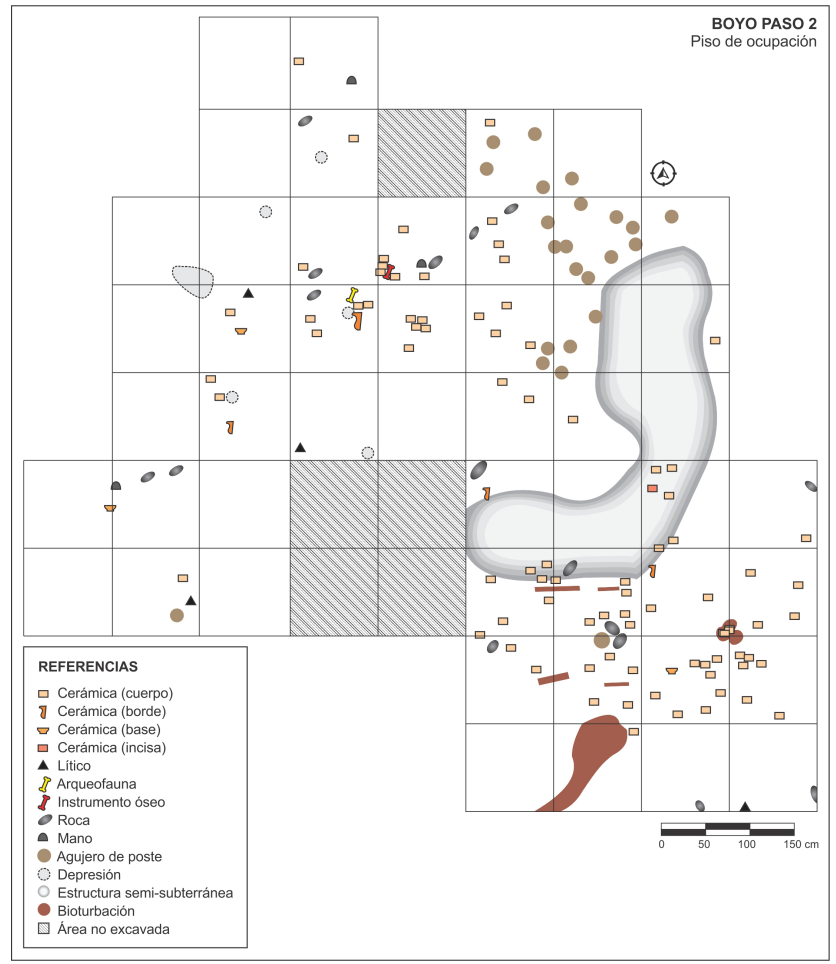

Figura 2. Planta del piso de ocupación superior de Boyo Paso 2.

Plan view of the upper archaeological floor from Boyo Paso 2.

tecnológicas y tipológicas se utilizaron aquellas piezas enteras o con fracturas poco relevantes, las cuales fueron clasificadas tentativamente en subgrupos tipológicos. También se empleó información de piezas que presentaban fracturas de importancia, pero solo de aquellos atributos no afectados por las mismas. En estas últimas, las fracturas se describieron de acuerdo con Weitzel et al. (2014), intentando obtener información sobre las causas probables de rotura de los instrumentos.

La adscripción funcional de las puntas de proyectil a lanza, dardo o flecha se basó en el peso (Fenenga 1953), intentando dilucidar el contexto social de uso en el marco de los modelos de movilidad y subsistencia tardíos. En este sentido, se asumió que las puntas que no superan los 4 gr formaron parte de flechas, 4-20 gr dardos y 20-70 gr lanzas arrojadizas, implicando distintos acercamientos a las presas y formas de organización social. También se utilizó el análisis de función discriminante a partir de dimensiones lineales de las puntas de proyectil, siendo en este caso el ancho máximo la medida considerada discriminante (Shott 1997). La forma de enmangue y las características de los astiles fue inferida sobre la base de la información recuperada de los pedúnculos y/o sectores basales, siguiendo los criterios de Hocsman (2010) y Knecht (1993).

Finalmente, la performance de los sistemas de armas fue evaluada a partir del análisis de diferentes aspectos que hacen a la efectividad de los mismos, como la capacidad de dañar órganos vitales, durabilidad, alcance, precisión, versatilidad para la captura de presas, posibilidades de recuperación, y otras (Christenson 1997; Ellis 1997; Hughes 1998; Loendorf et al. 2015; Lyman et al. 2009; Ratto 1989). Estas categorías fueron exploradas a través del estudio de los diseños (Bleed 1986), las frecuencias de artefactos, propiedades de las materias primas y modos de uso.

\section{Resultados}

El conjunto registró gran variabilidad de diseños, que fueron agrupados en cinco subgrupos tipológicos (Tablas 1, 2 y 3 ).

\section{A.1. Puntas de proyectil con pedúnculo diferenciado, aletas entrantes y limbo triangular corto de tipo isósceles}

Las medidas oscilan entre 27,57 y $16 \mathrm{~mm}$ (desvío estándar 4,74 mm). Poseen en promedio un ancho de $12,82 \mathrm{~mm}$, un espesor de $3,62 \mathrm{~mm}$ y un peso de 0,85 gr. Todas tienen pedúnculos de sección transversal biconvexa, con un ancho máximo de 4,83 $\mathrm{mm}$. Las formas base utilizadas para su manufactura fueron en su mayoría lascas de ópalo y calcedonia sin modificaciones 
Tabla 1. Principales características de las puntas de proyectil líticas enteras o con fracturas poco relevantes recuperadas en Boyo Paso 2.

Main characteristics of the complete or nearly complete lithic projectile points from Boyo Paso 2.

\begin{tabular}{|c|c|c|c|c|c|c|c|c|}
\hline Ejemplar Nro. & $\begin{array}{l}\text { Subgrupo } \\
\text { tipológico }\end{array}$ & Longitud (mm) & Ancho (mm) & Espesor (mm) & Peso (gr) & $\begin{array}{l}\text { Modulo Espesor } \\
\text { Pedúnculo }\end{array}$ & Materia Prima & Tipo de fractura \\
\hline Bp2-pp1 & A1 & 26,16 & 16 & 4,28 & 1,13 & 1,5 & $\begin{array}{l}\text { Calcedonia } \\
\text { Traslúcida }\end{array}$ & --- \\
\hline Bp2-pp2 & A1 & 18,19 & 13,28 & 4,23 & 0,81 & 1,68 & Calcedonia & $\begin{array}{c}\text { Acanaladura de } \\
\text { impacto; curvada }\end{array}$ \\
\hline Bp2-pp3 & A1 & 19,15 & 15,95 & 3,12 & 0,81 & --- & Calcedonia & $\begin{array}{c}\text { Acanaladura de } \\
\text { impacto; curvada en } \\
\text { el pedúnculo }\end{array}$ \\
\hline Bp2-pp4 & A1 & 20,67 & 9,9 & 4,9 & 0,79 & 1,51 & Calcedonia & --- \\
\hline Bp2-pp6 & A1 & 16,5 & 9 & 3,3 & 0,51 & 2,02 & Calcedonia & -- \\
\hline Bp2-pp7 & $\mathrm{A} 1$ & 27,77 & 12,54 & 4,57 & 1,04 & 2,35 & Calcedonia & --- \\
\hline Bp2-pp9 & $\mathrm{A} 1$ & 20,69 & 11,87 & 3 & 0,56 & 1,85 & Ópalo & Curvada \\
\hline Bp2-pp10 & Preforma & 23,45 & 14,2 & 6,1 & 1,66 & --- & Calcedonia & Curvada \\
\hline Bp2-pp11 & Preforma & 29,5 & 13,5 & 4,71 & 1,53 & --- & Calcedonia & -- \\
\hline Bp2-pp13 & A2 & 44,7 & 18,89 & 3,36 & 0,71 & 1,84 & $\begin{array}{l}\text { Calcedonia } \\
\text { Traslúcida }\end{array}$ & --- \\
\hline Bp2-pp14 & A1 & 24,65 & 13,23 & 4,3 & 0,88 & 1,93 & Calcedonia & $\begin{array}{l}\text { Acanaladura de } \\
\text { impacto }\end{array}$ \\
\hline Bp2-pp16 & A 3 & 12,84 & 10,74 & 2,85 & 0,32 & 1,33 & Ópalo & $\begin{array}{c}\text { Acanaladura de } \\
\text { impacto }\end{array}$ \\
\hline Bp2-pp22 & A1 & 22,76 & 10,9 & 3,7 & 0,75 & --- & Calcedonia & $\begin{array}{c}\text { Curvada en el } \\
\text { pedúnculo y ápice }\end{array}$ \\
\hline Bp2-pp24 & Preforma & 18,45 & 13,45 & 5,2 & 1,05 & --- & Calcedonia & --- \\
\hline Bp2-pp28 & A1 & 19,43 & 12,36 & 3,18 & 0,62 & --- & Indeterminada & $\begin{array}{c}\text { Curvada en el } \\
\text { pedúnculo }\end{array}$ \\
\hline Bp2-pp29 & $\mathrm{A} 1$ & 16 & 10 & 2 & 0,26 & --- & Calcedonia & --- \\
\hline Bp2-pp34 & Preforma & 20,15 & 14,5 & 6,82 & 1,63 & -- & Calcedonia & Curvada \\
\hline Bp2-pp35 & $\mathrm{A} 1$ & 25,2 & 16,5 & 5,45 & 1,86 & 2,2 & Indeterminada & --- \\
\hline
\end{tabular}

Tabla 2. Principales características de las puntas de proyectil líticas fracturadas recuperadas en Boyo Paso 2.

Main characteristics of the broken lithic projectile points from Boyo Paso 2.

\begin{tabular}{|c|c|c|c|c|c|c|}
\hline Ejemplar Nro. & Porción & Subgrupo & Ancho (mm) & Espesor (mm) & Materia prima & Tipo de fractura \\
\hline Bp2-pp5 & Fragmento de limbo basal & A1 & 13,12 & 3,45 & Calcedonia & Curvada; derivada \\
\hline Bp2-pp8 & Fragmento de limbo & A1 & 11,65 & 3,15 & Calcedonia & Curvada \\
\hline Bp2-pp12 & Fragmento de limbo basal & A1 & 13,32 & 4,16 & Calcedonia Traslúcida & Curvada en el limbo \\
\hline Bp2-pp15 & Fragmento de limbo & Indeterminada & 11,45 & 3,55 & Calcedonia & Curvada \\
\hline Bp2-pp17 & Fragmento de pedúnculo & Indeterminada & 4 & 2,05 & Calcedonia & Curvada \\
\hline Bp2-pp18 & Fragmento de limbo basal & A1 & 13,5 & 3,62 & Ópalo & Curvada en el limbo \\
\hline Bp2-pp19 & Fragmento de limbo & Indeterminada & 12,75 & 2,41 & Indeterminada & Curvada \\
\hline Bp2-pp21 & Fragmento de limbo basal & A1 & 16,09 & 3 & Indeterminada & Curvada; derivada \\
\hline Bp2-pp23 & Fragmento de limbo basal & A1 & 9 & 2,25 & Calcedonia & Curvada en el limbo \\
\hline Bp2-pp25 & Fragmentos de limbo basal & B1 & 25 & 5,26 & Cuarzo & Curvada en el limbo; derivada \\
\hline Bp2-pp26 & Fragmento de limbo basal & A2 & 15,3 & 2,1 & Calcedonia Traslúcida & Curvada en el limbo \\
\hline Bp2-pp27 & Fragmento de limbo basal & A1 & 15,65 & 3,78 & Calcedonia & Curvada en el limbo \\
\hline Bp2-pp30 & Fragmento de limbo basal & A1 & 12,55 & 2,96 & Calcedonia & Curvada en el limbo \\
\hline Bp2-pp31 & Fragmento de limbo & Indeterminada & 13,71 & 3,3 & Indeterminada & Curvada \\
\hline Bp2-pp32 & Fragmento de ápice & Indeterminada & 8,85 & 3,15 & Calcedonia & Curvada \\
\hline
\end{tabular}


Tabla 3. Principales características de los fragmentos de puntas de proyectil óseas (Subgrupo C.1.) de Boyo Paso 2.

Main characteristics of the fragments of bone projectile points (Subgroup C.1.) from Boyo Paso 2.

\begin{tabular}{ccccc}
\hline Ejemplar Nro. & Porción & Hueso soporte & Taxón & Observaciones \\
\hline BP2-29 & $\begin{array}{c}\text { Fragmento sección } \\
\text { mesial-lateral del } \\
\text { limbo }\end{array}$ & $\begin{array}{c}\text { Astilla de hueso } \\
\text { largo }\end{array}$ & $\begin{array}{c}\text { Macrovertebrado } \\
\text { indet. }\end{array}$ & $\begin{array}{c}\text { Fragmento termoalterado con } \\
\text { huellas de formatización }\end{array}$ \\
\hline BP2-56 & Fragmento limbo & $\begin{array}{c}\text { Astilla de hueso } \\
\text { largo }\end{array}$ & $\begin{array}{c}\text { Macrovertebrado } \\
\text { indet. }\end{array}$ & $\begin{array}{c}\text { Fragmento termoalterado con } \\
\text { huellas de formatización }\end{array}$ \\
\hline BP2-78 & Fragmento lateral \\
del limbo & $\begin{array}{c}\text { Astilla de hueso } \\
\text { largo }\end{array}$ & $\begin{array}{c}\text { Macrovertebrado } \\
\text { indet. }\end{array}$ & $\begin{array}{c}\text { Fragmento termoalterado con } \\
\text { huellas de formatización }\end{array}$ \\
\hline BP2-82 & $\begin{array}{c}\text { Fragmento } \\
\text { de pedúnculo } \\
\text { rectangular con } \\
\text { aserrado marginal }\end{array}$ & $\begin{array}{c}\text { Astilla de hueso } \\
\text { largo }\end{array}$ & $\begin{array}{c}\text { Macrovetebrado } \\
\text { indet. }\end{array}$ & $\begin{array}{c}\text { Fragmento termoalterado con } \\
\text { huellas de formatización }\end{array}$ \\
\hline
\end{tabular}

previas, de un tamaño y espesor similar al de las puntas líticas terminadas. Esta particularidad se hace visible en las piezas BP2-pp2 y BP2-pp23, con curvaturas demasiado marcadas, presentando incluso bulbos de percusión y restos de corteza. Existen divergencias entre los ejemplares en cuanto a la formatización, ya que algunas piezas presentan retoques unifaciales o marginales y otras bifaciales extendidos, pero siempre se mantiene el estándar en cuanto a forma y tamaño (Figura 3a). Las piezas BP2-pp9 y BP2-pp2, si bien evidencian que han sido reactivadas y/o retrabajadas con otros fines, no perdieron las características dimensionales y formales del diseño original. Cuatro ejemplares muestran señales de stress y/o pérdida abrupta de material -i.e. acanaladura de impacto- asociado a su utilización como proyectiles (sensu Weitzel et al. 2014). Cuatro preformas pueden ser incluidas dentro de este subgrupo. Total de puntas correspondientes a este subgrupo: 24.

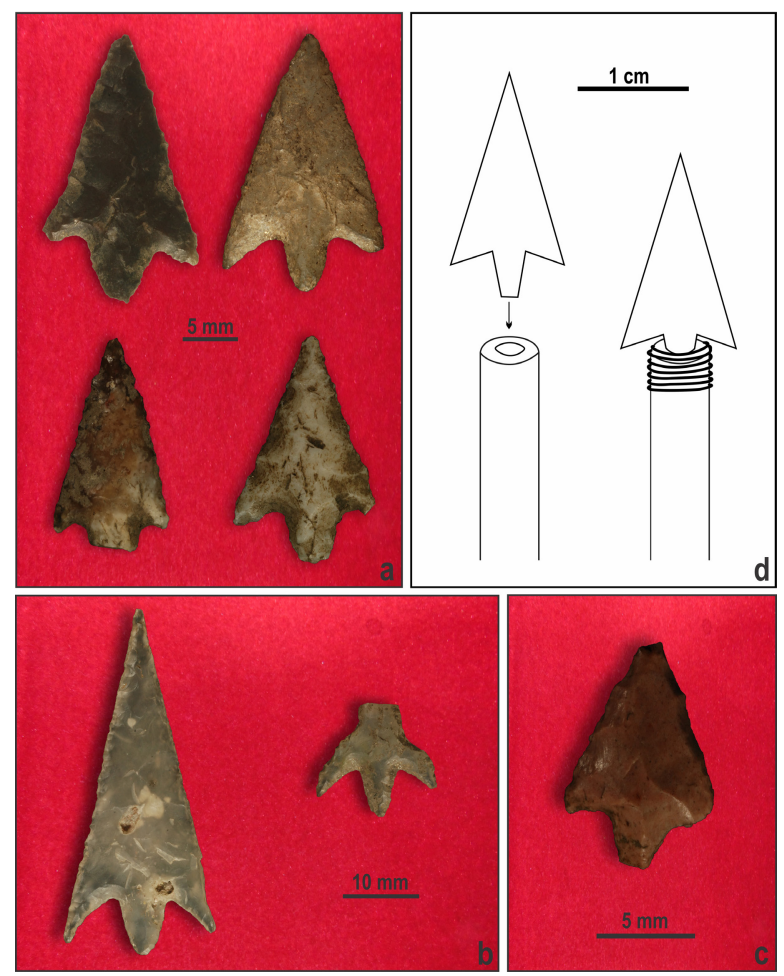

Figura 3. Subgrupos tipológicos de puntas de proyectil con pedúnculo diferenciado, aletas y limbo triangular: (a) Subgrupo tipológico A.1. (b) Subgrupo tipológico A.2. (c) Subgrupo tipológico A.3. (d) Esquema del sistema de enmangue descrito en el texto.

Typological subgroups of projectile points with triangular-shaped blade, contracted stems and barbed shoulders: (a) Typological subgroup A.1. (b) Typological subgroup A.2 (c) Typological subgroup A.3. (d) Schematic model of the hafting method described in the text. 
A.2. Puntas de proyectil con pedúnculo diferenciado, aletas entrantes y limbo triangular alargado de tipo isósceles

Se trata de dos piezas cuyas características no responden a las enunciadas para el subgrupo A.1. (Figura 3b). El limbo de BP2-pp26 fue reciclado en un perforador que se encuentra fragmentado, conservando la morfología del pedúnculo y el ancho máximo de las aletas. Las medidas del ejemplar entero (BP2-pp13) son 44,7 mm de largo máximo, 18,89 mm de ancho, 3,36 $\mathrm{mm}$ de espesor y 1,93 gr de peso. El pedúnculo es de sección transversal biconvexa, con un ancho máximo de 5,37 mm. Los dos ejemplares están elaborados en calcedonia translucida y presentan retoques bifaciales extendidos, con un acabado muy cuidado en lo relativo al mantenimiento de la delgadez y la simetría en las secciones longitudinal y transversal.

\section{A.3. Punta de proyectil con pedúnculo diferenciado, aletas entrantes y limbo triangular corto de tipo equilátero}

Se trata de un cabezal de tamaño muy pequeño, de $12,84 \mathrm{~mm}$ de longitud, 10,64 mm de ancho y 0,31 gr de peso, elaborado con micro-retoques marginales y retoques extendidos directamente sobre una lasca de ópalo rosado, que incluso presenta la curvatura original de la misma (Figura 3c). El ápice muestra una pequeña acanaladura de impacto, producto de su uso como proyectil. El pedúnculo es de sección biconvexa, con un ancho de 3,61 mm.

\section{B.1. Punta de proyectil de limbo triangular, apedúnculada con base escotada}

Se trata de un fragmento basal elaborado en cuarzo, con un largo estipulado de aproximadamente 38 $\mathrm{mm}, 5,25 \mathrm{~mm}$ de espesor, $25 \mathrm{~mm}$ de ancho y un peso estimado de $4 \mathrm{gr}$ (Figura 4a), este último determinado en base a la fórmula de Shott (1997). La forma base fue una lasca tamaño mediano, formatizada mediante retoques bifaciales extendidos. El ejemplar presenta una fractura curvada en el limbo y otra derivada en una de sus caras, lo que podría interpretarse como diagnóstica de impacto (Weitzel et al. 2014).

\section{C.1. Puntas de proyectil de hueso con limbo triangular de módulo alargado, aletas entrantes y pedúnculo rectangular con bordes aserrados}

Se recuperaron tres fragmentos de limbo y uno de pedúnculo (Figura 5a) que fueron asignados a este tipode
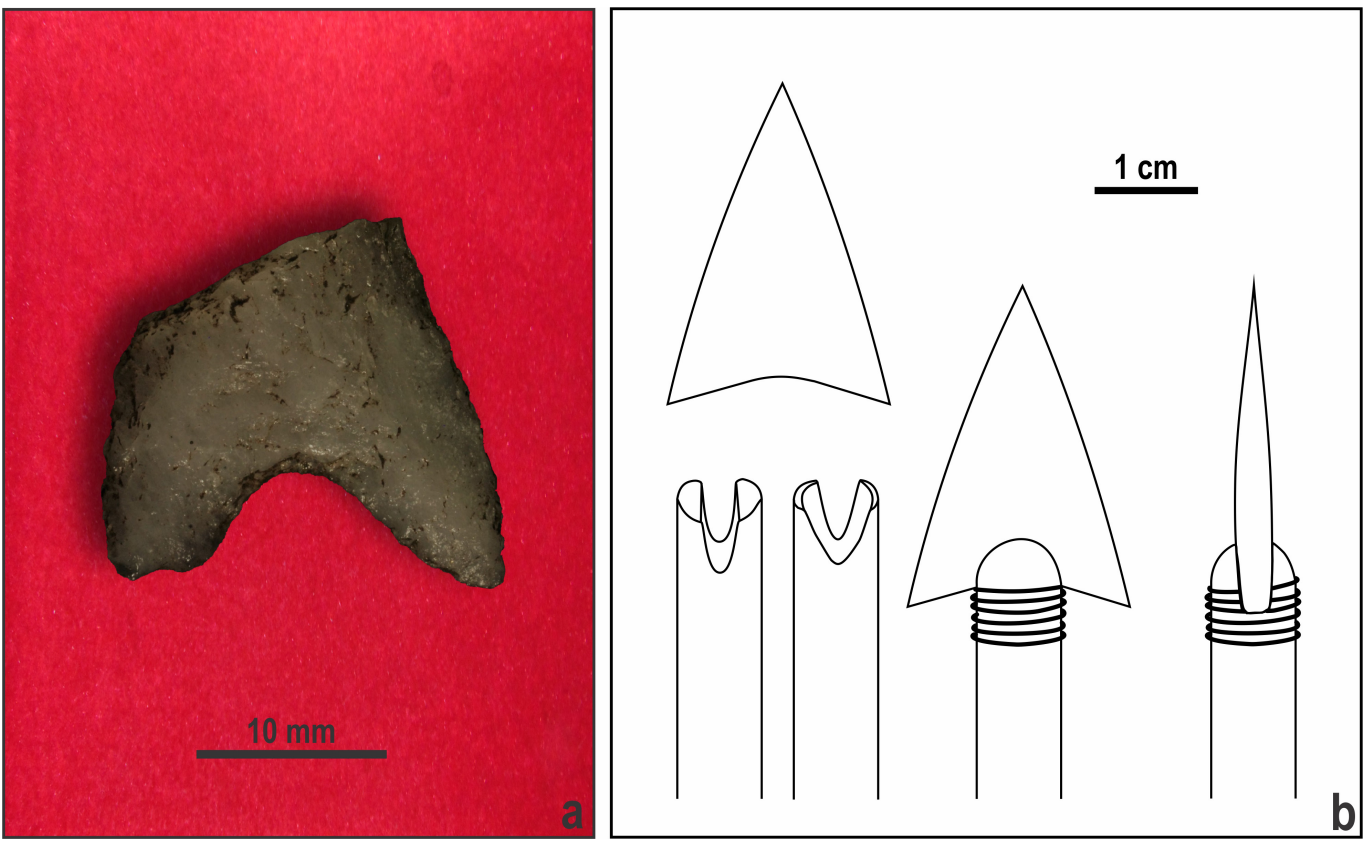

Figura 4. Punta de proyectil de limbo triangular, apedunculada con base escotada (Subgrupo tipológico B.1.): (a) Fragmento basal (BP2-pp25). (b) Esquema del sistema de enmangue descrito en el texto.

Unstemmed projectile point with a triangular-shaped blade and concave base (Subgroup B.1.): (a) Basal fragment (BP2-pp25). (b) Schematic model of the hafting method described in the text. 


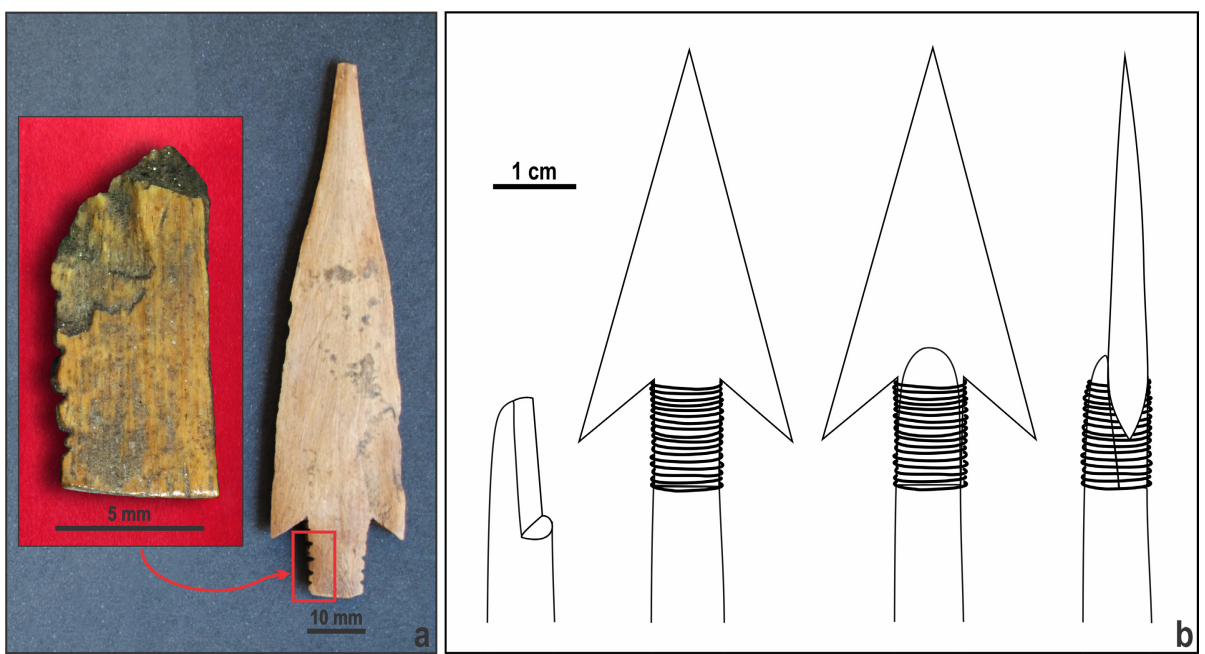

Figura 5. Punta de proyectil de hueso con limbo triangular de módulo alargado, aletas entrantes y pedúnculo rectangular con bordes aserrados: (a) fragmento de pedúnculo rectangular con bordes aserrados (BP2-82). (b) Esquema del sistema de enmangue descrito en el texto.

Projectile points with triangular-shaped blade, straight stems and barbed shoulders (Subgroup C.1.): (a) Fragment of a straight stem with serrated edge (BP2-82). (b) Schematic model of the hafting method described in the text.

puntas, recurrentes en la región serrana, con ejemplares enteros que promedian los $62,1 \mathrm{~mm}$ de largo, $14,6 \mathrm{~mm}$ de ancho, 2,6 de espesor y 2-4 gr de peso (Medina et al. 2014; Pastor et al. 2005; Rivero y Pauttassi 1999). Los fragmentos se encuentran termoalterados, presentando rastros de formatización por abrasión y pulido.

El ancho máximo y el peso de las piezas sugirieron el uso de al menos dos sistemas técnicos destinados a capturar presas. Los subgrupos A.1., A.2., A.3. y C.1. formaron parte de sistemas de armas impulsados por arcos, dado que por su poco peso requieren mayor velocidad para incrementar su efectividad y capacidad de penetración (Blitz 1988; Hughes 1998; Churchill 1993). En este sentido, las características de los pedúnculos de los subgrupos A.1., A.2. y A.3., sugieren la utilización de astiles livianos e intercambiables, de enchufes con hoyuelos que facilitaban el rápido reemplazo por un nuevo cabezal una vez recuperado el astil (Figura 3d). Estas flechas de diseño modular (sensu Bleed 1986) y enmangues no reforzados, coexistían con sistemas de enmangues más complejos que involucraban pedúnculos de mayor grado de elaboración y aserrado marginal para asegurar una firme atadura al astil, como es el caso de las puntas de hueso del subgrupo C.1 (Figura 5b).

La punta de proyectil apedúnculada B.1. formaría parte de un subgrupo que comparte muchas características con conjuntos tempranos ca. 4000-2000 AP de las Sierras de Córdoba (González y Crivelli 1978; Menghin y González 1954; Rivero 2009), de donde pudo ser reclamada. Se trata de una pieza con superficie y ancho relativamente grandes, que se enmangaba a un astil con enchufe de ranura simple tanto en "U" como en "V" de tamaño proporcionalmente mayor a los anteriormente considerados (Figura 4b), y que por su peso es compatible con sistemas arrojadizos de tipo propulsor, en donde este tipo de proyectiles tienen buena performance de vuelo, alcance y precisión (Pastor et al. 2005). Proyectiles con estas características pierden menos energía cinética con la distancia, lo que les confiere mayor poder de penetración (Hutchings y Brüchert 1997) y al utilizar cabezales de mayor tamaño pueden causar heridas más grandes (Christenson 1997), siendo óptimos para capturar animales de porte significativo. Sin embargo, su precisión puede ser menor a la de las flechas debido a su menor velocidad (Blitz 1988; Cattelain 1997; Churchill 1993; Hughes 1998; Pastor et al. 2005).

\section{Discusión}

La tecnología lítica y ósea del período Prehispánico Tardío de las Sierras de Córdoba ha sido caracterizada como expeditiva, dominada por artefactos informales que se elaboraban con materias primas disponibles en las inmediaciones de los sitios y se descartaban luego de finalizadas las tareas (Balena et al. 2018; Medina et al. 2014; Pastor 2007; Rivero y Pastor 2004). Sin embargo, el alto grado de formatización de las puntas de proyectil de Boyo Paso 2, elaboradas con materias primas que requirieron mayores esfuerzos de aprovisionamiento y manufactura, permite sostener que los grupos tardíos también desarrollaron artefactos más costosos, estandarizados y confiables (sensu Bleed 1986) para cumplir efectivamente con la captura de presas animales y/o violencia interpersonal. 
La dominancia del subgrupo A.1. en el registro arqueológico de Boyo Paso 2, con una frecuencia de $63 \%$, sugiere que estos cabezales fueron elementos comunes en los equipamientos de caza utilizados cotidianamente para abatir diversos animales, como también los más utilizados y descartados (Loendorf et al. 2015; Lyman et al. 2009; Politis et al. 2013). De esta manera, su presencia en los pisos de ocupación puede ser explicada con la realización in situ de actividades relacionadas con la reparación de armas luego de las cacerías, en donde se recambiaban cabezales y se aprovechaban astiles. Algunos de ellos también pudieron haber ingresado en asociación al cuerpo de alguna presa, sobre todo cuando las características de sus pedúnculos y enmangues no reforzados sugieren que quedaban retenidos en la carne cuando el astil era recuperado. Sin embargo, es llamativa, al menos desde una perspectiva occidental (Politis et al. 2013), la frecuencia de piezas completas o con daños que no parecen severos. Una explicación posible se asocia a la existencia de conductas orientadas a aprovisionar lugares (Medina et al. 2014), abandonando artefactos explotados por debajo de su potencial y/o preformas para ser utilizados nuevamente en futuras ocupaciones.

La identificación de una punta triangular del subgrupo B.1. puede ser interpretada como un caso de reclamación (sensu Schiffer 1987), en donde los pobladores se reapropiaron de esta pieza en algún contexto cazador-recolector más temprano y lo llevaron a Boyo Paso 2 donde finalmente quedó depositada. Argumenta a favor de este aserto la ausencia en el sitio de lascas de cuarzo asociadas a la realización de retoques bifaciales extendidos, más frecuentes en contextos arcaicos ca. 4000-2000 AP (ver Rivero 2009 y referencias allí citadas). Sin embargo, la reiterada documentación de este tipo de proyectiles en sitios tardíos (Balena et al. 2018) es indicativo de que el propulsor y el arco podrían haberse implementado simultáneamente para la caza de animales de gran porte como el guanaco (Lama guanicoe), aunque el uso del primero estaba en declinación frente a la efectividad del segundo. La presencia de descripciones etnohistóricas y de ganchos de propulsor óseos coexistiendo con proyectiles propulsados con arcos en contextos contemporáneos y/o coloniales tempranos de regiones vecinas, refuerza esta posibilidad (Sacur Silvestre et al. 2013; Swanton 1938).

Los subgrupos A.2 y C.1., con aletas agudas y módulos alargados de escaso espesor, cumplen con criterios de diseños de armas utilizadas para la violencia física efectiva (Loendorf et al. 2015), sin perjuicio de que otros cabezales hayan sido utilizados con el mismo propósito. Incluso, quizás eso explique por qué son menos abundantes en el registro de Boyo Paso 2 y otros sitios contemporáneos (Politis et al. 2013). La capacidad de estos diseños para perforar la caja torácica, corazón y pulmones, a los que se suman aletas con alta capacidad de retención y ataduras firmes para el caso de C.1., favorecen a que el proyectil no pueda ser fácilmente removido de la herida y produzca mayores daños internos en la persona herida, imposibilitando a la vez que el proyectil puede ser reutilizado (Christenson 1997; Hughes 1998; Loendorf et al. 2015). Existen casos etnográficos en donde lógicas y cabezales similares a C.1. son utilizados para cazar en ambientes boscosos, básicamente porque las flechas una vez en el blanco dificultan la huida de la presa e incrementan la magnitud de la hemorragia al enmarañarse con la vegetación (Griffin 1997; Politis et al. 2013). Sin embargo, la documentación de restos humanos a escasos kilómetros de Boyo Paso 2 con claros indicios de muerte producida por proyectiles armados con cabezales óseos similares a los aquí descritos (Pastor et al. 2012), así como otros publicados por González (1943), Fabra et al. (2015) y Weyenberg (1880), refuerza la hipótesis funcional aquí propuesta. De esta manera, la aparición ca. 1500 AP de armas diseñadas para la defensa y el ataque es indicativo de que durante el período Prehispánico Tardío se incrementó significativamente la violencia física interpersonal respecto a momentos anteriores. $\mathrm{La}$ preocupación por la violencia también quedó plasmada en el protagonismo que asumen las escenas de conflicto armado y/o despliegue de armas en algunos paneles de arte rupestre (Pastor 2012; Rivero y Recalde 2011). El hallazgo de cabezas trofeos o de cuerpos sin cabeza puede ser interpretado en el mismo sentido (Berberián 1984; González 1949; Paulotti 1943).

\section{Conclusión}

El estudio arqueológico del período Prehispánico Tardío de las Sierras de Córdoba durante décadas asumió que la incorporación de prácticas agrícolas rápidamente derivó en una mayor dependencia de los cultivos y en la sedentarización de los grupos en poblados compuestos por viviendas semi-subterráneas o casas-pozos (Berberián 1984; González 1943; Laguens y Bonnín 2009; Serrano 1945). Sin embargo, la ausencia de arquitectura permanente y la baja inversión en infraestructura agrícola, así como el resto de la evidencia contextual, impiden sostener que la agricultura haya sido la estrategia económica de mayor jerarquía y justificar un sedentarismo estricto (Medina et al. 2014; 2016; Medina et al. 2018). En cambio, la evidencia sugiere que las sociedades tardías desarrollaron una economía mixta que combinaba prácticas agrícolas con aportes significativos de caza-recolección, en donde la flexibilidad fue uno de los rasgos definitorios (Medina et al. 2014). La cerámica, el maíz y otros cultígenos fueron extensivamente utilizados, pero en el marco de una estrategia de subsistencia diversificada que buscaba incrementar la 
productividad por unidad de tiempo y espacio, e incluía un alto grado de movilidad a lo largo del ciclo anual (Medina et al. 2016; Medina et al. 2018; Pastor et al. 2012). De este modo, la caza-recolección continuó cubriendo una parte importante de la dieta, mientras que la agricultura fue estacionalmente interrumpida cuando los recursos silvestres de mayor rendimiento se encontraban disponibles en otros puntos del paisaje. El desarrollo de un nicho económico de amplio espectro, en consecuencia, se acompañaba de un grado variable de movilidad residencial, momentos de dispersión/ agregación de los grupos co-residentes y procesos estacionales de abandono de las prácticas productivas para abastecerse de recursos de caza y recolección, con antecedentes que pueden rastrearse hasta el Holoceno Medio (Medina et al. 2016; Pastor et al. 2012).

Dentro del modelo actualmente propuesto, los asentamientos residenciales a cielo abierto como Boyo Paso 2 reflejaban la concentración estival de un conjunto de unidades domésticas para realizar actividades agrícolas, recolectar, y capturar pequeños animales, así como para procesar, almacenar y consumir sus productos (Medina et al. 2014; Pastor et al 2012). El grupo coresidente, una vez finalizadas las tareas agrícolas y de recolección, se dispersaba hacia las pampas de altura para capturar ungulados silvestres y mantener la fluidez sociopolítica de la cual dependían. De este modo, los grupos tardíos hicieron un uso estacional y diversificado del paisaje, en donde las unidades domésticas o familiares constituyeron pequeños núcleos relativamente autónomos de producción, consumo y de ocupación del espacio, que se agregaban o dispersaban ajustándose a las cambiantes circunstancias del entorno (Medina et al. 2014; Pastor et al. 2012).

Es en este marco donde la diversidad de puntas de proyectil recuperadas en Boyo Paso 2, junto con las distintas formas de enmangue, la adopción generalizada del arco como forma de propulsión, el empleo selectivo de rocas y la incorporación de materias primas óseas, se convierte en una pieza clave para discutir aspectos fundamentales del proceso sociocultural tardío asociados con la intensificación económica y de las relaciones sociales. En este sentido, las características de los sistemas de armas son interpretadas como un indicador arqueológico de que la captura de vertebrados no era una simple actividad complementaria (Escola 2002). Por el contrario, integraría junto al cultivo y la recolección un sistema económico de tipo mixto, en donde conjuntos faunísticos dominados por ungulados silvestres y pequeños animales con huellas antrópicas de consumo argumentan a favor de este aserto (Medina et al. 2019). En este contexto, las puntas de proyectil impulsadas con arcos habrían jugado un rol crucial para abatir presas pequeñas y medianas-grandes con radios de captura distintos, situación coherente con un período donde se diversificaron las actividades de subsistencia y se incrementaron las tensiones sociales, con pruebas osteológicas concretas de violencia interpersonal.

El uso de proyectiles livianos también posibilitó adecuar la técnica de caza y/o defensa en función de las variaciones estacionales en el tamaño de los grupos, incrementando la autonomía económica y política de las familias. En otras palabras, el arco y la flecha permitieron cazar en forma individual sin necesariamente requerir la cooperación o ayuda mutua de varios cazadores (Bettinger 2013), facilitando que unidades sociales mínimas colonicen nuevos terrenos cultivables y/u ocupen estacionalmente espacios vacíos para la explotación de recursos silvestres. Esto no implicaba que no existieran cacerías grupales (Pastor et al. 2012), sino que finalizadas las cosechas y las tareas de almacenaje cada familia podía desarrollar estrategias individuales sin depender de la toma de decisiones de grupos más grandes. Por otro lado, creaba la oportunidad de una defensa individual efectiva, incrementando la autosuficiencia política de las familias y restringiendo el liderazgo institucionalizado a la duración del evento que involucraba agregación social y/o coresidencia, una situación consistente con el escenario de fragmentación política descripto en los documentos del tiempo de la conquista (González Navarro 2009).

Para finalizar, una de las principales propiedades que surgen al observar los sistemas de armas del componente prehispánico tardío de Boyo Paso 2 es la variedad de cabezales y materias primas utilizadas en su confección. Esto denota, como mínimo, la amplitud del nicho económico, pero particularmente la preocupación por minimizar la falla en la captura de las presas de las cuales dependían. Los datos obtenidos, de esta manera, apuntan hacia la existencia de una fuerte componente de cazarecolección entre los grupos tardíos, que junto con el cultivo, regían el ciclo de movilidad anual. Incluso es difícil estimar en base a la información disponible la importancia de la agricultura frente a la caza-recolección, al menos a partir de unos pocos cotiledones de Phaseolus spp. y fitolitos de Zea mays. En tal sentido, las sociedades tardías de las Sierras de Córdoba compartieron rasgos con otras poblaciones del Viejo y Nuevo Mundo en donde la incorporación de cultivos dio lugar a múltiples combinaciones entre cazarecolección y agricultura (Layton et al. 1991), demostrando la fluidez entre los dos modos de producción.

Agradecimientos: Este trabajo es el resultado de investigaciones financiadas por la Agencia Nacional de Promoción Científica y Tecnológica (PICT-2016- 2010677). Deseamos expresar nuestro agradecimiento a Robin Torrence, Lucía Yebra, Salomón Hocsman, Robert Bettinger, Diego Gobbo, Norma Ratto, Patricia Escola y a los tres revisores anónimos, quienes aportaron bibliografía y comentarios que ayudaron a mejorar la calidad del manuscrito. 


\section{Referencias Citadas}

Aschero, C. 1975. Ensayo para una Clasificación Morfológica de Artefactos Líticos Aplicada a Estudios Tipológicos Interpretativos. Informe inédito presentado al CONICET, Buenos Aires.

Aschero, C. 1983. Ensayo para una Clasificación Morfológica de Artefactos Líticos. Apéndices A y B. Apunte inédito para la Cátedra de Ergología y Tecnología. Facultad de Filosofía y Letras, Universidad de Buenos Aires, Buenos Aires

Aschero, C. y G. Martínez 2001. Técnicas de caza en Antofagasta de la Sierra, Puna Meridional Argentina. Relaciones de la Sociedad Argentina de Antropología 26:215-241.

Balena, I., G. Heider y M. Medina 2018. Tecnología lítica entre las sociedades del Período Prehispánico Tardío (Sierras de Córdoba, Argentina). Mundo de Antes 12 (2), en prensa.

Banegas, A. J. Gómez Otero, S. Goye y N. Ratto 2014. Cabezales líticos del Holoceno Tardío en Patagonia Meridional: diseños y asignación funcional. Magallania 42 (2):155-174.

Berberián, E. 1984. Potrero de Garay: Una entidad sociocultural tardía de la región serrana de la Provincia de Córdoba (Rep. Argentina). Comechingonia 4:71-138.

Bettinger, R. 2013. Effects of the bow on social organization in Western North America. Evolutionary Anthropology 22:118-123.

Bleed, P. 1986. The optimal design of hunting weapons: maintainability or reliability. American Antiquity 51:737-747.

Blitz, J. 1988. Adoption of the bow in prehistoric North America. North American Archaeologist 9 (2):123-145.

Castro, S. 2017. Tecnología de caza en la cuenca alta del río Las Taguas (San Juan, Argentina). Análisis morfológico de proyectiles líticos del sitio ARQ-18 (8.900-790 AP). Cuadernos del Instituto Nacional de Antropología y Pensamiento Latinoamericano 26 (1):1-20.

Cattelain, P. 1997. Hunting during the Upper Paleolithic: Bow, spearthrower, or both? En Projectile Technology, editado por H. Knetch, pp. 213-240. Plenum Press, New York.

Christenson, A. 1997. Side-notched and unnotched arrowpoints. Asssesing functional differences. En Projectile Technology, editado por H. Knetch, pp. 131-142. Plenum Press, New York.

Churchill, S. 1993. Weapon technology, prey size selection and hunting methods in modern hunter-gatherer: implications for hunting in the Paleolithic and Mesolithic. En Hunting and Animal Exploitation in the Later Paleolithic and Mesolithic of Eurasia, editado por G. Peterkin, H. Bricker y P. Mellars, pp. 11-24. Archaeological Papers of the American Anthropological Association, New York.

Ellis, C. 1997. Factor influencing the use of stone projectile tips. An ethnographic perspective. En Projectile Technology, editado por H. Knetch, pp. 37-78. Plenum Press, New York.

Escola, P. 1991. Puntas de proyectil de contextos formativos: acercamiento tecno-tipológico a través de cuatro casos de análisis. Actas del Congreso Nacional de Arqueología Chilena, Tomo 2, pp. 175-184. Museo Nacional de Historia Natural, Santiago.

Escola, P. 2002. Caza y pastoralismo: un reaseguro para la subsistencia. Relaciones de la Sociedad Argentina de Antropología 27:233-245.

Escola, P. 2014. Proyectiles líticos en contexto de Arroyo Seco 2: algo más que una tecnología para la caza. En Estado
Actual de las Investigaciones en el Sitio Arqueológico Arroyo Seco 2 (Partido de Tres Arroyos, Provincia de Buenos Aires, Argentina), editado por G. Politis, M. Gutiérrez y C. Scabuzzo, pp. 229-275. Universidad Nacional de Centro de la Provincia de Buenos Aires, Tandil.

Fabra, M., C. González y S. Robin 2015. Evidencias de violencia interpersonal en poblaciones del piedemonte y las llanuras de Córdoba (Argentina) a finales del Holoceno tardío. Runa 36:5-27.

Fenenga, F. 1953. The weights of chipped stone points: A clue to their functions. Southwestern Journal of Anthropology 9 (3):309-323.

González, A. 1943. Arqueología del yacimiento indígena de Villa Rumipal (Pcia. de Córdoba). Publicaciones del Instituto de Arqueología, Lingüística y Folklore IV. Universidad Nacional de Córdoba, Córdoba.

González, A. 1949. Nota sobre la arqueología de Pampa de Olaen (Córdoba). Notas del Museo de La Plata. Tomo XIV. Antropología 56:463-503.

González Navarro, C. 2009 Autoridades étnicas en un contexto de desestructuración: Córdoba entre la fundación y la visita de Antonio Martines Luxan de Vargas. En Visita a las Encomiendas de Indios de Córdoba 1692-1693, editado por B. Bixio, C. González Navarro, R. Grana y V. Iarza, pp. 63-114. Editorial Brujas, Córdoba.

González, S. y E. Crivelli 1978. Excavaciones arqueológicas en el abrigo de Los Chelcos (Dpto. San Alberto. Córdoba). Relaciones de la Sociedad Argentina de Antropología 12:183212.

Griffin, B. 1997. Technology and variation in arrow design among the Agta of Northwestern Luzon. En Projectile Technology, editado por H. Knetch, pp. 267-286. Plenum Press, New York.

Heider G., D. Rivero y E. Baldo 2015. Rocas de uso arqueológico en Sierras Centrales. Fuentes de recursos líticos identificadas y potenciales en las provincias de Córdoba y San Luis, Argentina. Revista de Antropología del Museo de Entre Ríos 1 (2):55-72.

Hocsman, S. 2010. Cambios en las puntas de proyectil durante la transición de cazadores-recolectores a sociedades agropastoriles en Antofagasta de la Sierra (Puna Argentina). Arqueología 16:59-86.

Hughes, S. 1998. Getting to the point: evolutionary change in prehistoric weaponry. Journal of Archaeological Method and Theory 5 (4):345-408.

Hutchings, W. y L. Brüchert 1997. Spearthrower performance: Ethnographic and experimental research. Antiquity 71:890-897.

Knecht, H. 1993. Early Upper Paleolithic approaches to bone and antler projectile technology. En Hunting and Animal Exploitation in the Later Paleolithic and Mesolithic of Eurasia, editado por G. Peterkin, H. Bricker y P. Mellars, pp. 33-47. Archaeological Papers of the American Anthropological Association, New York.

Laguens, A. y M. Bonnín 2009. Sociedades Indígenas de las Sierras Centrales. Arqueología de Córdoba y San Luis. Editorial de la Universidad Nacional de Córdoba, Córdoba. 
Layton, R., R. Foley y E. Williams 1991. The transition between hunting and gathering and the specialized husbandry of resources. Current Anthropology 32:255-274.

Loendorf, C., L. Simon, D. Dybowski, M. Woodson, R. Plumlee, S. Tiedens y M. Withrow 2015. Warfare and big game hunting: flaked-stone projectile points along the middle Gila River in Arizona. Antiquity 89:940-952.

Luti, R., M. Bertrán de Solís, F. Galera, N. Müller de Ferreira, M. Berzal, M. Nores, M. Herrera y J. Barrera 1979. Vegetación. En Geografía Física de la Provincia de Córdoba, editado por J Vázquez, R. Miatello y M. Roqué, pp. 297-368. Editorial Boldt, Buenos Aires.

Lyman, R., T. VanPool y M. O'Brien 2009. The diversity of North American projectile-point types, before and after the bow and arrow. Journal of Anthropological Archaeology 28:1-13.

Marcellino, A., E. Berberián y J. Pérez 1967. El yacimiento arqueológico de Los Molinos (Dpto. Calamuchita, Córdoba). Publicaciones del Instituto de Antropología 26:1-68.

Martínez, J. y D. Bozzuto (eds.) 2011. Armas Prehispánicas. Múltiples Enfoques para su Estudio en Sudamérica. Fundación de Historia Natural Felix de Azara, Buenos Aires.

Medina, E., L. López L y N. Buc 2018. Bone Tool and tuber processing: a multi-proxy approach at Boyo Paso 2, Argentina. Antiquity 92:1040-1055.

Medina, M., N. Buc, S. Pastor 2014. Intensificación y dinámica ocupacional en el periodo Prehispánico Tardío de las Sierras de Córdoba (Argentina): una aproximación desde el registro artefactual óseo. Chungara Revista de Antropología Chilena 46 (1):73-90.

Medina, M., M. Campos, N. Ávila, E. Soibelzon y F. Fernández 2019. Animal food during the Late Prehispanic Period at Sierras of Córdoba (Argentina). A zooarchaeological view from Boyo Paso 2. Anthropozoologica, en prensa.

Medina M., S. Grill, A. Fernández y L. López 2017. Anthropogenic pollen, foraging and crops during Sierras of Córdoba Late Prehispanic Period (Argentina). The Holocene 27:1769-1780.

Medina, M., S. Pastor y E. Berberián 2014. Es gente fazil de moverse de una parte a otra. Diversidad en las estrategias de subsistencia y movilidad prehispánicas tardías (Sierras de Córdoba, Argentina). Complutum 25 (1):73-88.

Medina, M., S. Pastor S y A. Recalde 2016. The archaeological landscape of late prehispanic mixed foraging and cultivation economy (Sierras of Córdoba, Argentina). Journal of Anthropological Archaeology 42:88-104.

Menghín, O. y A. González 1954. Excavaciones arqueológicas en el yacimiento de Ongamira, Córdoba (Rep. Arg.). Nota preliminar. Notas del Museo de La Plata, T XVII, Antropología 67. Universidad Nacional de La Plata, La Plata.

Moreno, E. 2011. Tecnología de caza en la Quebrada de Antofalla, Departamento Antofagasta de la Sierra, Catamarca. Revista del Museo de Antropología 4:17-32.

Nelson, M.1991. The study of technological organization Advances in Archaeological Method and Theory 3:57-100.

Pastor, S. 2007. Arroyo Tala Cañada 1 (Valle de Salsacate). Espacio doméstico y productivo en el sector central de las Sierras de Córdoba
(Argentina) durante el Período Prehispánico Tardío (1000-300 AP) Arqueología 14:41-74.

Pastor, S. 2012. Arte rupestre, paisaje y tensión social: un caso de estudio en Córdoba, Argentina. Revista Chilena de Antropología 26:7-32.

Pastor, S., M. Medina, A. Recalde, L. López y E. Berberián 2012 Arqueología de la región montañosa central de Argentina. Avances en el conocimiento de la historia prehispánica tardía. Relaciones de la Sociedad Argentina de Antropología 37:89-112.

Pastor, S., D. Rivero y E. Pautassi 2005. Los sistemas de armas de las comunidades agroalfareras de Córdoba: una aproximación arqueológica y experimental. Actas del XIII Congreso Nacional de Arqueología Argentina, Tomo IV, pp. 253-266. Córdoba.

Paulotti, O. 1943. Tipos de inhumación de los antiguos habitantes de las fuentes del río Tercero (Dpto. de Calamuchita, Prov. De Córdoba). I Congreso de Historia Argentina del Norte y Centro, Tomo I, pp. 239-266. Córdoba.

Pautassi, E. y D. Rivero 1999. La configuración de los sistemas de armas y su relación con la estructura de los recursos en la cuenca del río San Antonio (Dpto. de Punilla, Pcia. de Córdoba). Actas del XII Congreso Nacional de Arqueología Argentina Tomo III, pp. 517-520, La Plata.

Politis, G., A. González Ruibal, A. Hernando y E. Beserra Coelho 2013. Etno-arqueologia do descarte de flechas entre os Awá-Guajá da Floresta Amazônica brasileira. En Estudos sobre os Awá, Caçadores-Coletores em Transição, editado por A. Hernando y E. Beserra Coelho, pp. 131-154. EDUFMA, São Luís-Maranhão.

Ratto, N. 1989. Eficacia funcional y técnicas de caza. Shincal 1:45-52.

Ratto, N. 2013. Diversidad de tecnologías de caza en la Puna transicional de Chaschuil (Dpto. Tinogasta, Catamarca). Comechingonia 17 (2):85-103.

Restifo, F. 2013. Tecnología de caza durante el Holoceno Temprano y Medio en la Puna de la Provincia de Salta (República Argentina): patrones de variación y procesos de cambio. Comechingonia 17:59-84.

Rivero, D. 2009. Ecología de Cazadores-Recolectores del Sector Central de las Sierras de Córdoba (Rep. Argentina). BAR, International Series 2007, Oxford.

Rivero, D. y S. Pastor 2004. Sistemas de producción lítica de las comunidades productoras de alimentos de las sierras de Córdoba. Análisis de tres conjuntos de la Pampa de Achala. En Terceras Jornadas de Arqueología Histórica y de Contacto del Centro Oeste de la Argentina y Seminario de Etnohistoria. Cuartas Jornadas de Arqueología y Etnohistoria del Centro Oeste del país, editado por M. Bechis, pp. 67-78. Universidad Nacional de Río IV, Río Cuarto.

Rivero, D. y A. Recalde 2011. El uso del arco en la guerra durante el Prehispánico tardío de las Sierras de Córdoba. En Armas Prehispánicas: Múltiples Enfoques para su Estudio en Sudamérica, editado por J. Martínez y D. Bozzuto, pp. 151-171. Fundación de Historia Natural Felix de Azara, Buenos Aires.

Sacur Silvestre, R., N. Buc, A. Acosta y D. Loponte 2013. Estrategias de captura de presas y sistemas de armas de los cazadoresrecolectores que habitaron el Humedal del Paraná Inferior: una aproximación experimental y arqueológica. Comechingonia $17: 27-57$ 
Schiffer, M. 1987. Formation Process of the Archaeological Records. University of New Mexico Press, Albuquerque.

Serrano, A. 1945. Los Comechingones. Serie Aborígenes Argentinos I. Instituto de Arqueología, Lingüística y Folklore de la Universidad Nacional de Córdoba, Córdoba.

Shott, M. 1997. Stones and shafts redux: the metric discrimination of chipped-stone dart and arrow points. American Antiquity 62 (1):86-101.

Swanton, J. 1938. Historic Use of the spear-throw in Southwestern North America. American Antiquity 3 (4):356-358.
Vigna, M., I. González y C. Weitzel 2014. Los cabezales líticos de la microrregión del río Salado bonaerense, Argentina. Diseños e historias de vida. Intersecciones en Antropología 15:55-69.

Weitzel, C., N. Flegenheimer y M. Colombo 2014. Breakage patterns on fishtail projectile points: experimental and archaeological cases. Ethnoarchaeology 6 (2):81-102

Weyenbergh, H. 1880 Alt-indianische werkzeuge, pfeilspitzen u. dgl. Verhandlungen der Berliner Gesselschaft für Anthropologie, Ethnologie und Urgeschichte XII:366-374.

\section{Nota}

${ }^{1}$ La identificación de las materias primas se realizó por comparación macroscópica con materiales previamente identificados a partir de cortes petrográficos por la geóloga Dra. Irene Hernando (CONICET-CIG; Facultad de Ciencias Naturales y Museo, Universidad Nacional de La Plata). Los datos referentes a su distribución, disponibilidad y calidad provienen de Heider et al.
(2015). De este modo, el cuarzo y la calcedonia fueron considerados rocas inmediatamente disponibles en los alrededores de Boyo Paso 2, aunque esta última en escasa frecuencia en comparación a la abundancia del cuarzo, incrementando los costos de su obtención. El ópalo, por otra parte, proviene de fuentes localizadas a $100 \mathrm{~km}$ al norte del sitio, por lo que se define como una roca no local. 
